\title{
Design of Gateway of 4G and ZigBee Network Fusion Technology Based on Intelligent Street Light System
}

\author{
Yongjun Zhang, Xuan Wang \\ State Key Laboratory of Information Photonics and Optical Communications \\ Beijing University of Posts and Telecommunications, 100876, China
}

Keywords: ZigBee;4G;ARM Cortex-A9;Gateway; intelligent street light

\begin{abstract}
In order to solve the problem of poor switching between wireless communication signals in traditional streetlight systems, the intelligent streetlight gateway based on ZigBee wireless sensor network and $4 \mathrm{G}$ network module is designed. ${ }^{[1,2]}$ The gateway uses Freescale's ARM Cortex-A9 embedded processor as the master chip, ARM Linux real-time operating system, using ZigBee and 4G fusion of communication technology to achieve the formation of wireless networks and the connection with the Internet, and gives the wisdom of street lamps Gateway hardware component structure and software implementation process. ZigBee module to complete the data acquisition, 4G module with the remote WEB server data transmission to meet the needs of lighting control and remote monitoring. ${ }^{[3]}$ The experimental results show that the gateway is reliable and stable, and is superior to wired gateway and GPRS gateway in packet loss rate and time response, and is suitable for application in intelligent streetlight system.
\end{abstract}

\section{Introduction}

With the development of communication technology, computer technology and network technology, intelligent street lights gradually become a node of the future wisdom of the city. The function of traditional street light is single, can't realize the supervision and control management to the street lights, and the maintenance cost and the failure rate are high. The intelligent street lights use ZigBee technology and 4G data transmission technology to communicate. ${ }^{[4]}$ The communication network will be collected data access to the Internet, through a friendly man-machine interface to achieve real-time monitoring of the entire area lights, allowing users to remotely monitor the scene status and control of street lighting equipment. The degree of intelligence is improved significantly. ZigBee is a low-speed short-range transmission of wireless network protocol, is ideal for intelligent street lights between the various equipment communications. ${ }^{[5]} 4 \mathrm{G}$ as a mainstream wireless communication technology, its highspeed, low cost, low power, self-configuration and flexible network structure. It is very suitable for data transmission. $4 \mathrm{G}$ module and ZigBee wireless sensor network docking to achieve relatively simple, just through the RS232 can be.

\section{Intelligent street light gateway design structure}

The system mainly includes two aspects: sensor nodes and core controller. The sensor node is mainly responsible for the intelligent street lights of the information parameters of the collection, and through the ZigBee protocol to send the data to the core controller. The core controller will distinguish the different nodes according to the different address codes, and the result of data analysis through the $4 \mathrm{G}$ protocol and TCP / IP protocol conversion, to complete the connection with the internet and to transmit to Remote server; remote server to achieve real-time monitoring of the work status of the street. ${ }^{[6]} \mathrm{PC}$ terminal equipment is supported on the system management. Intelligent street light gateway design structure are shown in fig.1.

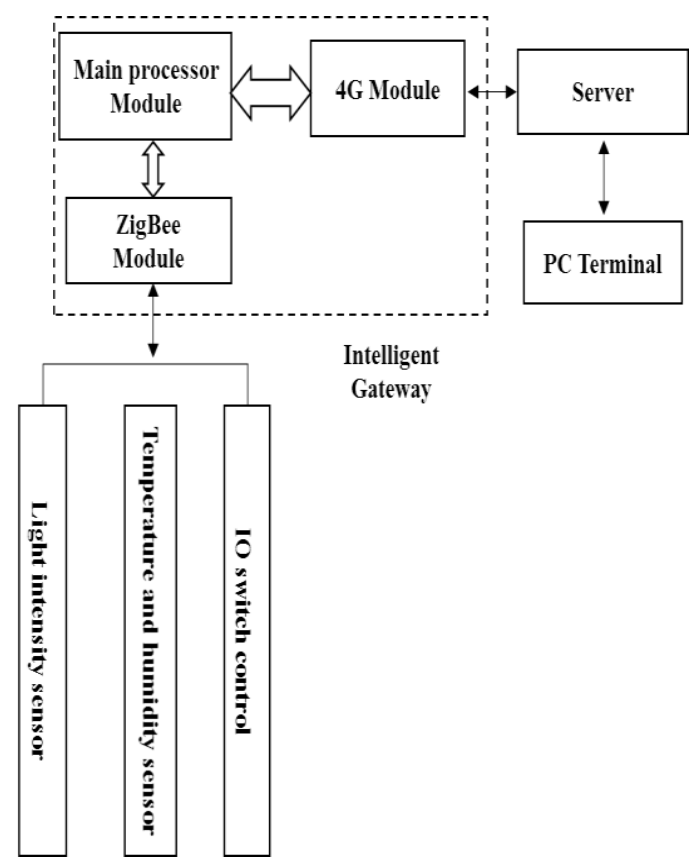

fig.1: Intelligent street light gateway design structure

ZigBee networking module. In the intelligent street light system, the use of ZigBee wireless communication technology will be composed of smart devices star network. Star network is a radial structure, network commands and data are transmitted through the coordinator. The 
communication between the terminal equipment is also forwarded through the coordinator. The terminal device is either the starting point of communication, or the end of the communication. As a network coordinator, communication ZigBee expansion module can be the beginning or end in the home gateway, and can be responsible for the sub-sensor node communication management, dynamic networking and data transmission. Terminal nodes include street lights in the internal network of LED switches, temperature and humidity sensors, light sensors, radios cameras and so on.

In order to distinguish the data of different sensor nodes and the reliability of the data transmission. When sending a message, use a custom protocol, a total of eight, the first sent Short address, the second used to determine whether the data is received or switch control system, and returns two bytes as the response information of the slave machine, it is mean that communication is successful. The last two bits of the protocol guarantee communication reliability for CRC checksum. The format of the message protocol is shown in Table I.

\begin{tabular}{|l|l|l|l|}
\hline $\begin{array}{l}\text { Short } \\
\text { Address }\end{array}$ & $\begin{array}{l}\text { Switch } \\
\text { Control }\end{array}$ & $\begin{array}{l}\text { Data } \\
\text { Bit }\end{array}$ & $\begin{array}{l}\text { CRC } \\
\text { Checksum }\end{array}$ \\
\hline 2 Bytes & 2 Bytes & 2 Bytes & 2 Bytes \\
\hline
\end{tabular}

Table I: A custom protocol in ZigBee

4G networking module. 4G wireless module uses Siemens SIM7300C as a chip. It has been embedded with TCP / IP protocol stack, to support $4 \mathrm{G}$ communication, with RS232 serial port, SIM card interface. Selecting Siemens SIM7300C as $4 \mathrm{G}$ module is due to its strong anti-interference, cheap, high quality, convenient serial communication and its suitability for street lighting system design requirements. The docking between 4G module and ZigBee wireless sensor network achieve relatively simple, just through the RS232.

Main processor module is the core of the entire intelligent street light control system, the design uses Freescale's ARM Cortex-A9 chip as the master chip, the choice of Siemens SIM7300C as 4G chip. The processor integrates a rich peripheral resource such as LCD controller, USB Host, NAND controller, BUS controller, interrupt control, power control, memory control, UART, SPI and GPIO, through external expansion memory, serial port, USB Interface and JTAG debugging interface etc. the hardware circuit block diagram shown in fig. 2 .

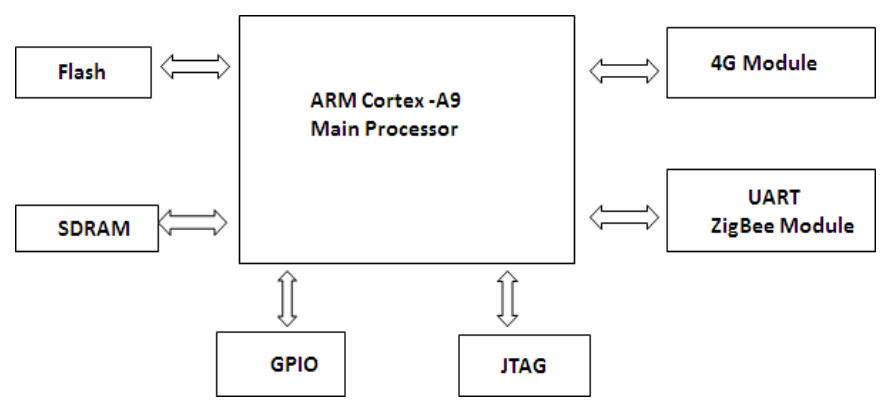

fig.2: Arm cortex -A9 system block diagram

\section{The design and structure of gateway including software and hardware}

\subsection{The composition of gateway.}

The intelligent streetlight gateway adopts the modular design scheme, which is composed of the hardware driver layer, the operating system layer, the network protocol layer and the application layer. The hardware driver layer describes the Zigbee module and 4G module driver in the gateway node; the operating system layer transplanted the ARM Linux realtime operating system kernel; the network protocol layer ported Zigbee and $4 \mathrm{G}$ protocol stack, Zigbee and $4 \mathrm{G}$ protocol bidirectional conversion. The program layer ported the embedded Web server software on the Linux kernel. The protocol conversion diagram of $4 \mathrm{G}$ module and ZigBee module is shown in fig. 3

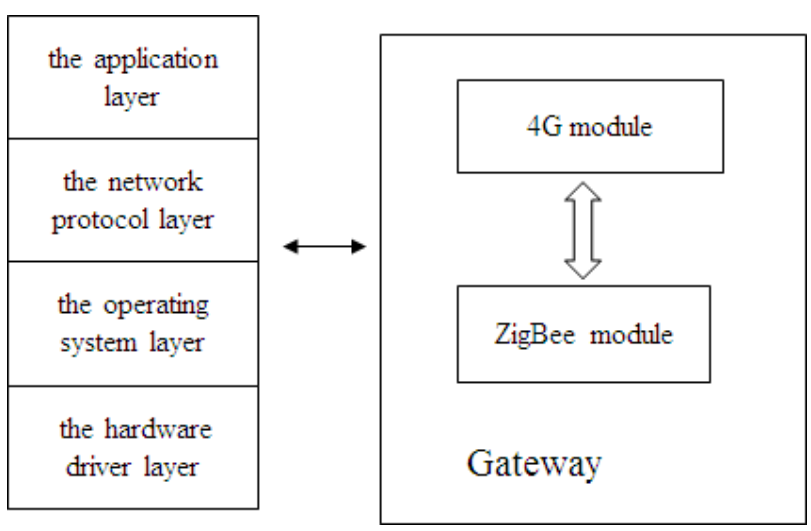

fig.3: The protocol conversion diagram of $4 \mathrm{G}$ module and ZigBee module

The processes of communications. The intelligent street light gateway needs to be parsed above the transport layer. Usually it connects two or more independent networks. After each packet is received, it is converted to another protocol format before forwarding. The Zigbee protocol stack consists of a series of hierarchical structure, including the physical layer, data link layer, network layer, application support layer and application layer, each layer for the next layer to provide services. The node address information and monitoring data to be transmitted by the acquisition node are sent to the gateway node in the form of Zigbee frame. Transferring the data through the physical media into the gateway, the first stack with Zigbee protocol stack to get the original data. Through TCP / IP protocol, package data is transferred to the Internet, and the gateway and the Internet connected. In order to realize the monitoring and control functions of the system, the design of the data frame structure is composed of the source address, the data pattern, the destination address, the data length, the data information and the checksum, and so on The data information, the checksum, and so on. The data information field is divided into the direction bit, function type and data. Directional bit is divided into upstream and 
downstream: upstream transmission is the collected data, the downstream transmission is the control command.

The processes of program. Main program process of intelligent gateway is shown in fig.4. After the hardware power system is started, the application is initialized, the gateway starts listening to the network, and the incoming gateway enters the interrupt status. First the program needs to judge whether Zigbee data. If it is Zigbee data, according to the ZigBee protocol to process the data and then forwarded to the $4 \mathrm{G}$ module, the gateway returns to the listening state; if not Zigbee data, the gateway to determine whether the $4 \mathrm{G}$ data, if it is $4 \mathrm{G}$ protocol processing data and then forwarded to Zigbee module, and return to the monitoring state; if it is unknown data, the gateway to discard directly, then return to monitor the network status.

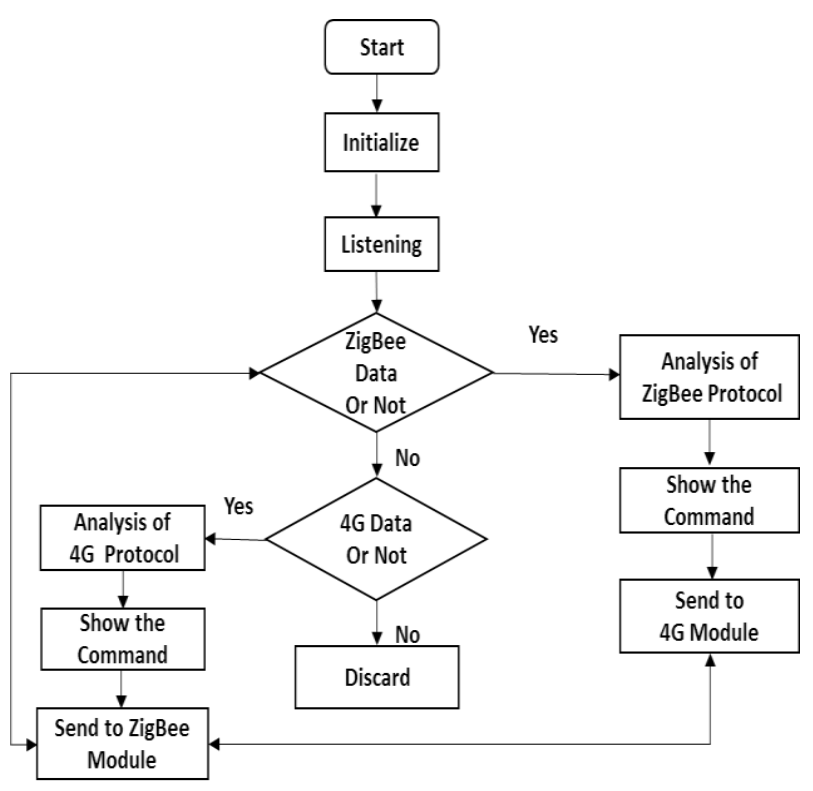

fig.4: The program process of intelligent gateway

\section{Flow estimation algorithm and experimental analysis}

\subsection{Flow estimation algorithm Based on Gateway}

In order to reduce the packet loss and shorten the packet transmission delay, this paper uses the traffic estimation algorithm to make the intelligent gateway to maximize the use of wireless link resources. The Pre ACK mechanism can increase the amount of data sent by the sender at the unit time. However, sending the Pre ACK frequently will cause the sender to send a large number of packets in a short time to cause network congestion. Therefore, it is necessary to establish a mechanism to control the Pre ACK Of the transmission rate. In this paper, a variable - threshold is used to limit the sending rate of the Pre ACK. In the Local buffer, the packets on the right side of the threshold can send a Pre ACK and the left will not send a Pre ACK. The threshold determines the transmission rate of the sender to a certain extent and controls the network traffic. It will Local-buffer is divided into two parts, the ideal link state, when the threshold is fixed, the data sender's transmission rate can be said to be constant, that is, the channel bandwidth is constant. But the actual network of each data will go through different networks, and with the establishment and closure of the connection, the emergence and recovery of congestion, the threshold should also be dynamic changes, so in order to adapt to the data in the two networks in the dynamic changes, it should also change the threshold dynamically. Based on the previous research results, this paper proposes to dynamically adjust the threshold based on the estimated available bandwidth of the link, so that each connection is fair and enjoy the bandwidth and maximize the use of wireless link resources.

Adjust the field value of each data channel, when receiving a Real ACK, calculate the ideal rate and actual rate. The difference between the smallest diff. According to the intelligent gateway to receive the Real ACK round trip delay to estimate the bandwidth, and accordingly, if the actual rate and the expected rate is very close, diff value is very small, you can think that the network is better, no congestion, the connection of the watermark Plus 1 ; if the actual rate is much smaller than the expected rate, diff value is larger, then the network is not good, keep the watermark value of the connection unchanged. Because Real ACK arrives at the smart gateway very quickly, the watermark value is rapidly increasing, thus facilitating the rapid convergence of the initial throughput of the TCP connection establishment.

$$
\begin{aligned}
& \text { Ideal Rate = base RTT } / \text { count } \\
& \text { Actual rate }=\text { current RTT } / \text { count } \\
& \text { Diff }=\text { ideal rate }- \text { actual rate }
\end{aligned}
$$

Where count is the number of backup packets sent to the receiving end in the Local buffer of the current TCP connection, base RTT represents the smallest RTT record in the Real ACK received by the smart gateway, and curr RTT represents the Real ACK of the current TCP connection Of the RTT value. When the watermark increases to a certain size, the TCP connection throughput may have converged to a limit, and then use the above strategy to adjust the watermark, there may be due to excessive growth of watermark lead to send the sender too fast, resulting in Network congestion. In order to avoid this situation, the watermark should be allowed to increase slowly, that is, by least squares to estimate the bandwidth available for the entire link, so as to avoid the impact of random factors on the throughput, so that the link bandwidth estimation becomes more accurate and reasonable.

\subsection{Performance analysis of the gateway}

Generally, There are several technical indicators influence on the test of smart Street light network, such as time delay, packet loss rate and other aspects. In order to verify the application performance of the gateway in the intelligent 
streetlight system, the following is tests' analysis of two aspects of the performance: Gateway packet loss changes with number of trials in the test, the gateway delay with the time to change the test.

The test of gateway's packet loss rate change with number of trials: It refers to the ratio which the number of packet lost and the proportion of the number of packets should be in unit time within the transmission process. In this test, the main influencing factors are network quality and signal attenuation. Intelligent street light gateway packet loss mainly includes two aspects, on the one hand, it is packet loss of ZigBee device node, on the other hand, it is the packet loss of communication between $4 \mathrm{G}$ module and the upper platform. In order to be as close to the actual use as possible, the gateway node receives data to make statistics of packet loss for every 1500 packet. The following is formula of the packet loss rate. It received 1500 packets in the test, the loss number is $n$, the packet loss rate for the formula 1 :

$$
\text { Loss }=\mathrm{n} / 1500 * 100 \%
$$

The statistical analysis of the experiment is shown in Fig. 3. The figure shows the packet loss rate with the gateway working time changes of the curve. In Fig. 3, it is probably 0.0009 in the packet loss rate of gateway consisting of Bluetooth networks and $4 \mathrm{G}$ networks. However, it is approximately 0.0003 that the packet loss rate of gateway consisting of ZigBee networks and $4 \mathrm{G}$ networks equals. In contrast to fig.5, the packet loss of gateway consisting of ZigBee networks and $4 \mathrm{G}$ networks is much lower than the packet loss of gateway consisting of Bluetooth networks and 4G networks. And also it is lower than the China Communications Standards Association. CCSA provides the maximum packet loss rate of 0.001 to meet the practical application requirements.

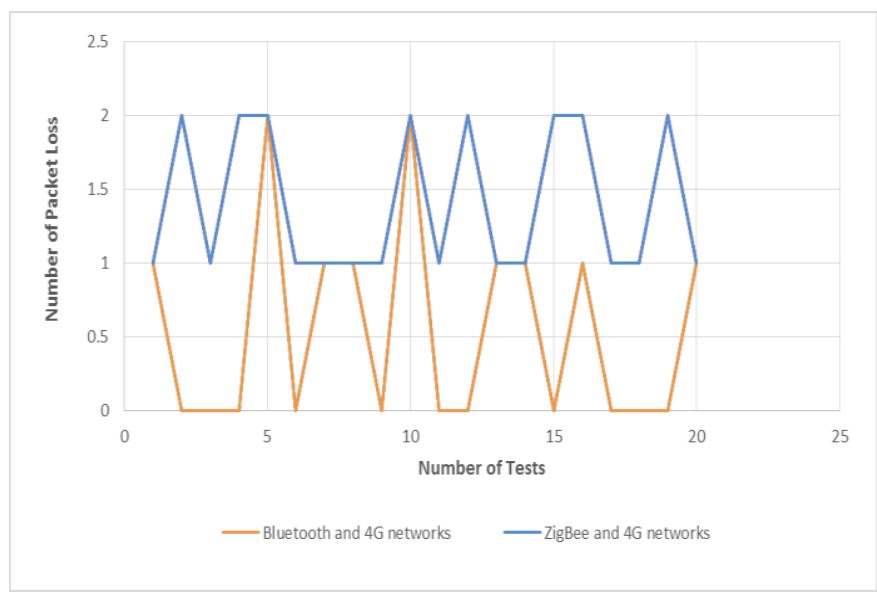

fig.5: The packet loss of gateway

The test of gateway delay change with time: At the beginning, the gateway of intelligent street light reads a complete data packet from the serial port, then transmit data. In this process, the gateway receives and sends 1500 packets. We make a contrast test between gateway made up of Bluetooth networks and $4 \mathrm{G}$ networks and gateway made up of ZigBee networks and $4 \mathrm{G}$ networks.

After analysis, the average forwarding delay time is $50 \mathrm{~ms}$ in gateway of ZigBee and $4 \mathrm{G}$ network, in comparison, the average forwarding delay time is $80 \mathrm{~ms}$ in gateway made up of Bluetooth networks and $4 \mathrm{G}$ networks, in fig.6. It can be seen that both average delays are much less than the CCSA standard of the upper limit of $100 \mathrm{~ms}$. The statistical analysis of the experiment is shown that the gateway of ZigBee and $4 \mathrm{G}$ network is better to meet the practical application requirements.

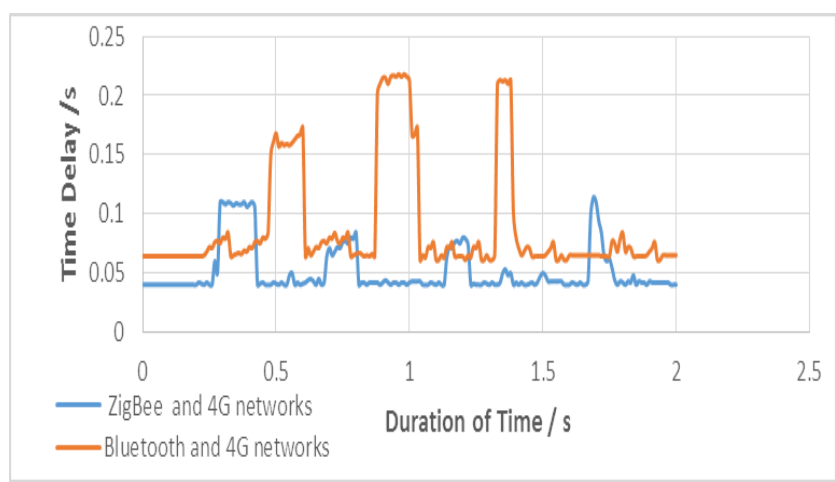

fig.6: The time delay of gateway

The test of average through put in gateway changes with time: This indicator describes the network equipment packet forwarding performance. That is, in the absence of packet loss conditions, the network equipment unit time to forward the limits of data packets.

The time interval between the completion of a complete packet start and the completion of the forwarding. The indicator is mainly by the gateway unpacking and packaging Packet, network transmission delay, related to the system to deal with real-time. The experimental results are shown in fig. 6

It is shown that the home gateway designed in this paper is basically in a stable state during the duty cycle.

\section{Conclusions}

It is shown that the intelligent street light gateway designed in this paper is basically stable. The simulation results show that the gateway of intelligent street light gateway designed in this paper has better performance in packet loss rate, delay time. It meets the performance requirements of the streetlight control system network, and achieves the stability of the streetlight control network, real-time and have good performance.

\section{References}

[1] Edward A. Lee, Cyber-Physical Systems - Are Computing Foundations Adequate, Position Paper for NSF Workshop On Cyber-Physical Systems: Research Motivation, Techniques and Roadmap, October 16 - 17, 2006 Austin, TX. 
[2] Wang Zhongjie, Cyber-physical Systems: A Survey, J. 14(2006)51-56.

[3] Drew Gislason, Zigbee Wireless Networking, Newnes, 2008.

[4] Ma Xiaojun, Intelligent lighting control system, Southeast university press, 2009.

[5] Sun Z, Wang W. "Investigation of cooperation technologies in heterogeneous wireless networks," Journal of Computer Systems, Networks and Communications, Vol 2010 (2010):1-12.

[6] Nathalie M., Symeon P., et al. "Combining Cloud and sensors in a smart city environment," EURASIP Journal on Wireless Communications and Networking, No. 247(2012), pp. 1-10. 